\title{
Uso de redes neurais artificiais na detecção de propagação de defeitos em dutos rígidos
}

\author{
Use of artificial neural networks in detection of propagation \\ of defects in pipelines rigid
}

Pinto, C.F.C ${ }^{1}$; Silva, R.R. ${ }^{1}$; Calôba, L.P. ${ }^{1}$; Soares, S. D. ${ }^{2}$

\author{
${ }^{1}$ Laboratório de Processamento de Sinais, Universidade Federal do Rio de Janeiro; Rio de Janeiro, Brasil \\ e-mail: carlos.fernando@lps.ufrj.br ; romeu@ romeu.eng.br ; caloba@1ps.ufrj.br \\ ${ }^{2}$ Centro de Pesquisa Leopoldo Américo Miguez de Mello, PETROBRAS; Rio de Janeiro, Brasil; \\ e-mail: sdama@petrobras.com.br
}

\begin{abstract}
RESUMO
O interesse no monitoramento de equipamentos em tempo real é crescente nos dias de hoje, visando a maior segurança de operação dos mesmos. O ensaio de Emissão Acústica vem sendo objeto de desenvolvimentos com o intuito de aplicação em diversos tipos de equipamentos, com destaque na inspeção de dutos rígidos e flexíveis. O presente trabalho apresenta a metodologia e os resultados obtidos de um estudo de aplicação do método de Emissão Acústica para detectar defeitos em propagação em dutos rígidos pressurizados, sendo um trabalho pioneiro nessa área de pesquisa. Para tal, foram confeccionados corpos de prova com defeitos inseridos artificialmente, empregando-se a técnica de ultrassom por Time-of-flight diffraction (TOFD) para acompanhar o crescimento dos defeitos no duto submetido a ensaio hidrostático. Os sinais resultantes foram divididos em classes de Propagação, Propagação Estável e Propagação Instável, e usados como dados de entrada na implementação de classificadores não lineares via Redes Supervisionadas do tipo Retropropagação do Erro. Os resultados atingiram acertos de classificação próximos a 86\%, comprovando a eficiência do método para as condições testadas até o presente momento.
\end{abstract}

Palavras-chave: Ensaios não destrutivos, Redes Neurais, Emissão Acústica.

\section{ABSTRACT}

The interest in monitoring equipment in real time is increasing in nowadays, mainly aiming the greater security of its operations. The Acoustic Emission (AE) testing has been the subject of developments with the aim of application in various types of equipment, especially in the inspection of rigid and flexible pipes. This paper presents the methodologies and results of a study of applying the method of Acoustic Emission to detect propagation in defects in pressurized rigid pipes, being a pioneering work in this area of research. In this way, specimens were manufactured with defects artificially inserted. These specimens were submitted to hydrostatic testing and the defect propagation was monitor by AE. The ultrasound by Time of Flight Diffraction (TOFD) was the technique chosen to monitor the defect growth. The AE resulting signals were divided into the classes No Propagation (SP), Stable Propagation (PE) and Unstable Propagation (PI) and used as inputs set in the implementation of nonlinear classifiers by error back propagation. The correct classification results reached close to $86 \%$, proving the efficiency of the method for the conditions tested in this job.

Keywords: Nondestructive testing (NDT), acoustic emission, neural networks

\section{INTRODUÇÃO}

No mundo moderno, há inegável importância da utilização dos Ensaios Não Destrutivos (END) na avaliação da integridade estrutural de equipamentos. No passado, poucos eram os ensaios aplicáveis, basicamente se restringindo ao Ensaio Visual, Líquidos Penetrantes, Partículas Magnéticas, Radiografia e Ultrassom. Dentre os ensaios não destrutivos atuais, destaca-se o método de Emissão Acústica (EA), o qual está baseado na 
detecção de fontes de sinais acústicos que são emitidas durante a propagação de descontinuidades e deformações plásticas acentuadas. Por ser um método qualitativo, o ensaio de emissão acústica não fornece as dimensões das descontinuidades, as quais são fornecidas por outros métodos de ensaios não destrutivos, como o ultrassom. Uma vez que só fornece indicações de descontinuidades ativas durante o carregamento das estruturas, um dos principais objetivos de sua aplicação é o monitoramento em tempo real de equipamentos.

Uma das principais linhas de pesquisa e desenvolvimento hoje na área de END é a utilização de ferramentas de processamento de sinais, imagens e reconhecimento de padrões para semi-automatização ou automatização de processos de detecção e classificação de defeitos em equipamentos. Diversos destes trabalhos vêm sendo desenvolvidos na última década com os sinais de ultrassom computadorizado e imagens radiográficas digitais [1-6].

Motivado pela importância da Emissão Acústica entre os métodos não destrutivos usados na inspeção de equipamentos, e a potencialidade de se implementar classificadores não lineares pelas técnicas de rede neurais, o presente trabalho tem como objetivo desenvolver classificadores não lineares de padrões, tendo como entrada os parâmetros dos sinais de EA, visando detectar o crescimento dos defeitos em dutos rígidos em três classes de sinais: Sem Propagação, Propagação Estável e Propagação Instável, as quais foram definidas pelo acompanhamento por ultrassom de crescimento de defeitos usando a técnica Time-of-flight diffraction (TOFD) simultaneamente à realização de ensaio hidrostático em corpos de prova (trecho de dutos). É importante destacar que o presente trabalho faz parte de um trabalho de dissertação de mestrado e de um projeto de trabalho de pesquisa realizado entre Petrobras/CENPES/TMEC, LPS/COPPE e LAMEF/UFRGS.

\section{ANÁLISE DOS ENSAIOS DE EMISSÃO ACÚSTICA}

\subsection{Materiais}

O material dos corpos de prova é aço API XL Grau 60, tendo estes 20 polegadas de diâmetro e 14,5 mm de espessura. Os tubos tiveram suas extremidades usinadas a fim de que fossem montados dois flanges para contenção do fluido de pressurização (vedação feita com o-rings de borracha). Foram usinadas trincas elípticas nas superfícies internas e externas dos tubos, cujas dimensões eram diferentes em cada ensaio. Foram realizados seis ensaios ao todo no projeto, porém, apresentam-se neste artigo apenas os dois últimos ensaios por se tratarem dos resultados mais conclusivos do projeto. Para ilustração, a Figura 1 contém um desenho esquemático típico do corpo de prova com a instrumentação utilizada para o monitoramento dos ensaios hidrostático.

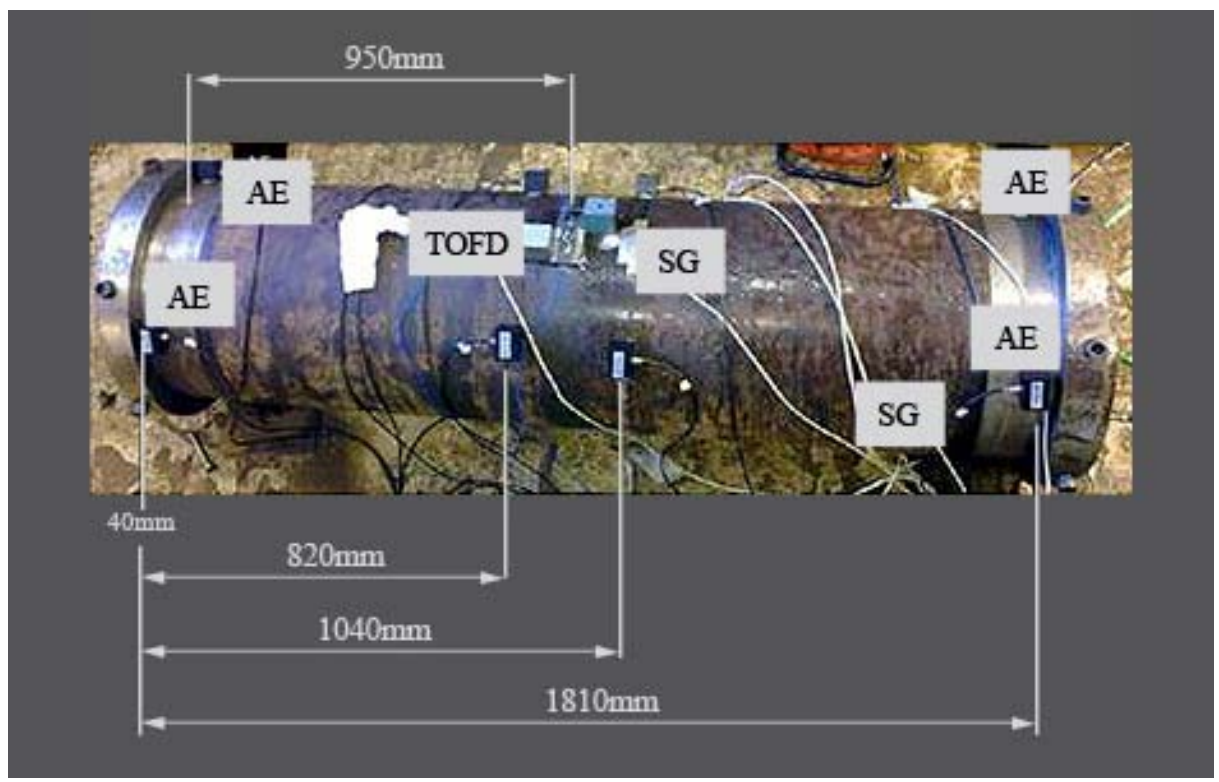

Figura 1: Esquema de posicionamento dos sensores próximos à região de presença da trinca interna na superfície do corpo de prova. Cotas em milímetros. 


\subsection{Aquisições dos parâmetros de emissão acústica}

Os sinais de emissão acústica foram adquiridos durante os ensaios de pressão hidrostática, utilizando o equipamento Disp 16C da PASA (Physical Acustic South America). Após a aquisição, os sinais foram processados no próprio equipamento, os parâmetros adquiridos são apresentados na Tabela 1 [1, 2].

Tabela 1: Parâmetros de Emissão Acústica coletados [1, 2]

\begin{tabular}{|c|c|}
\hline PARÂMETRO & DESCRIÇÃO \\
\hline Rise & Tempo do primeiro pico a ultrapassar o limiar de referência até o pico de amplitude máxima. \\
\hline Count & Número de vezes em que o sinal ultrapassa o limiar, dentro do valor definido de HDT. \\
\hline Energy & $\begin{array}{l}\text { Integral do valor absoluto, ou módulo, de cada pico da onda. Como se trata de um sinal temporalmente } \\
\text { discreto, somam-se os valores das amostras da forma de onda retificada. Cabe salientar que a } \\
\text { amplitude aqui se refere a uma medida de tensão/voltagem e não decibel. }\end{array}$ \\
\hline Duration & $\begin{array}{l}\text { Intervalo de tempo entre a primeira e a última ultrapassagem do limite, dentro do valor pré- } \\
\text { estabelecido de HDT. }\end{array}$ \\
\hline AMP & Amplitude máxima do sinal, em dB. \\
\hline A-Freq & Frequência Média: a definição é contagem sobre duração, [count/duration]. \\
\hline RMS & $\begin{array}{l}\text { Raiz da integral dos valores de tensão ao quadrado dividida pelo intervalo de tempo dado pela } \\
\text { diferença de tempo entre os limites superior e inferior de integração. }\end{array}$ \\
\hline ASL & Nível médio do sinal, em dB. \\
\hline PCNTS & $\begin{array}{l}\text { Número de picos até a amplitude máxima ser alcançada, sempre levando em consideração o } \\
\text { limiar de referência. }\end{array}$ \\
\hline R-Freq & $\begin{array}{l}\text { Frequência de reverberação: definida por: [contagem - contagem ao pico] / } \\
\text { [duração - tempo de subida]. }\end{array}$ \\
\hline I-Freq & Frequência de Iniciação (definida por: contagem ao pico / tempo de subida) \\
\hline Sig-Strength & $\begin{array}{l}\text { Intensidade ou potência do sinal: praticamente a definição de energia, porém com } \\
\text { coeficiente mais simples de sen(pi/4). }\end{array}$ \\
\hline ABS-Energy & $\begin{array}{l}\text { Energia Absoluta: quantidade real de energia sendo a unidade base pico Joule, definido pela } \\
\text { soma das amplitudes das amostras ao quadrado dividido pela impedância de } 10 \mathrm{kOhm} \text {. } \\
\text { É importante lembrar que este parâmetro é normalizado. }\end{array}$ \\
\hline Freq-PP1 & Primeira potência parcial. \\
\hline Freq-PP2 & Segunda potência parcial. \\
\hline Freq-PP3 & Terceira potência parcial. \\
\hline Freq-PP4 & Quarta potência parcial. \\
\hline C-Freq & $\begin{array}{l}\text { Centróide de frequência: não necessariamente a frequência central, correspondente ao centro do } \\
\text { espectro, mas uma frequência que leva em consideração “pesos” devido à magnitude e espalhamento. }\end{array}$ \\
\hline P-Freq & Frequência pico: componente de frequência de maior magnitude no espectro. \\
\hline
\end{tabular}

\subsection{Monitoramento do crescimento dos defeitos}

O crescimento destas trincas foi monitorado através de ensaios de ultra-som usando a técnica TOFD (Time of Flight Diffraction).

\subsection{Sincronização dos arquivos de emissão acústica e pressão}

Com o monitoramento da pressurização e do crescimento dos defeitos artificiais, além dos sinais de emissão acústica, que se encontram sempre em arquivos distintos, foi estabelecido um procedimento para sincronizálos, pois a atividade de emissão acústica está relacionada com o carregamento imposto e com a propagação do defeito. Os sinais de EA resultantes dos ensaios foram filtrados de maneira simples apenas para separar, por tempo de chegada, aqueles que proviam dos sensores próximos aos defeitos. 


\subsection{Separação dos sinais}

Após sincronização dos arquivos, os eventos de emissão acústica foram divididos nas classes SP (Sem Propagação) e Propagação (P), ou SP, PE (Propagação Estável) e Propagação Instável (PI).

\subsection{Pré-processamento dos dados}

Primeiramente, os dados que estavam inicialmente em formato de arquivo DTA foram convertidos em formato ASC II. Posteriormente, os dados foram normalizados adequadamente para melhor desempenho no treinamento das redes neurais.

\subsection{Análise de relevância dos parâmetros de emissão acústica}

Este critério mede a relevância de cada parâmetro de entrada do classificador, isto é, mede o quanto este influencia no desempenho da rede neural. O método consiste em procurar mudanças significativas na resposta do classificador, ao substituir os valores de cada parâmetro pelo valor médio [7].

A relevância $\mathrm{R}\left(\mathrm{X}_{\mathrm{i}}\right)$ da componente $\mathrm{X}_{\mathrm{i}}$ do vetor $\mathrm{X}$ pode ser calculada pela seguinte equação:

$$
R\left(X_{t}\right)=\frac{\sum_{j=1}^{p}\left\|y\left(x_{j}\right)-y\left(x_{j i}\right)\right\|^{2}}{P}
$$

$\mathrm{R}\left(\mathrm{X}_{\mathrm{i}}\right)$ : relevância da componente $\mathrm{x}_{\mathrm{i}}$ dos vetores de entrada $\mathrm{x}$;

$\mathrm{P}$ : número de padrões (treinamento ou teste);

$\mathrm{y}\left(\mathrm{x}_{\mathrm{j}}\right)$ : vetor de saída da rede neural para cada padrão i x de entrada apresentado;

$\mathrm{x}_{\mathrm{ji}}$ : vetor de entrada $\mathrm{x}_{\mathrm{j}}$, em que a i-ésima componente foi substituída pelo seu valor médio tomado sobre todos os vetores de entrada;

$\mathrm{y}\left(\mathrm{x}_{\mathrm{ji}}\right)$ : saída da rede para a entrada $\mathrm{x}_{\mathrm{ji}}$

\subsection{Elaboração dos algoritmos para desenvolvimento dos classificadores e avaliação de classificação}

Para implementação dos classificadores de padrões, foram implementados classificadores de padrões não lineares usando redes neurais artificiais [8,9].

Redes Neurais Artificiais (RNA) são estruturas inspiradas nas redes neurais biológicas. Seu comportamento se assemelha a capacidade de reconhecimento do cérebro humano, o qual funciona de maneira completamente diferente dos computadores digitais convencionais. Ele tem a capacidade de organizar suas estruturas, os neurônios, formando redes capazes de realizar operações (reconhecimento de padrões, percepção, controle motor e etc.) em menos tempo que o mais rápido computador da atualidade [8].

A ideia central das RNAs é combinar linearmente as entradas obtidas a partir dos parâmetros coletados, e modelar as saídas (resultado a ser atingido) como funções não lineares destes parâmetros [10]. Existe um amplo espectro de modelos de redes neurais, neste trabalho é aplicado o modelo chamado de Multilayer Feedfoward Networks.

A menor unidade de uma RNA é o neurônio, ele é responsável pelo processamento da informação e é formado por três elementos básicos, as sinapses, um somador e uma função de ativação, a Figura 1 apresentada um esquema desta estrutura.

De acordo com a Figura 2, as sinapses são responsáveis por associar a cada entrada $\mathrm{X}_{\mathrm{i}}$ um peso $\mathrm{W}_{\mathrm{i}}$, que determina o efeito da mesma sobre o neurônio. Estas são combinadas linearmente pelo somador e a função de ativação, geralmente uma função não linear, determina o nível de ativação do neurônio artificial. Estes são organizados em camadas e de acordo com a arquitetura utilizada, pode haver uma ou mais camadas. 


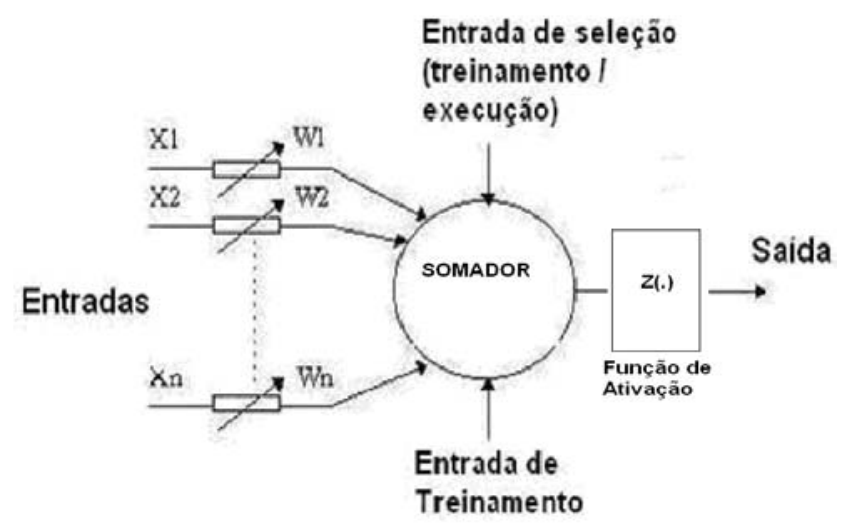

Figura 2: Modelo não linear de um neurônio.

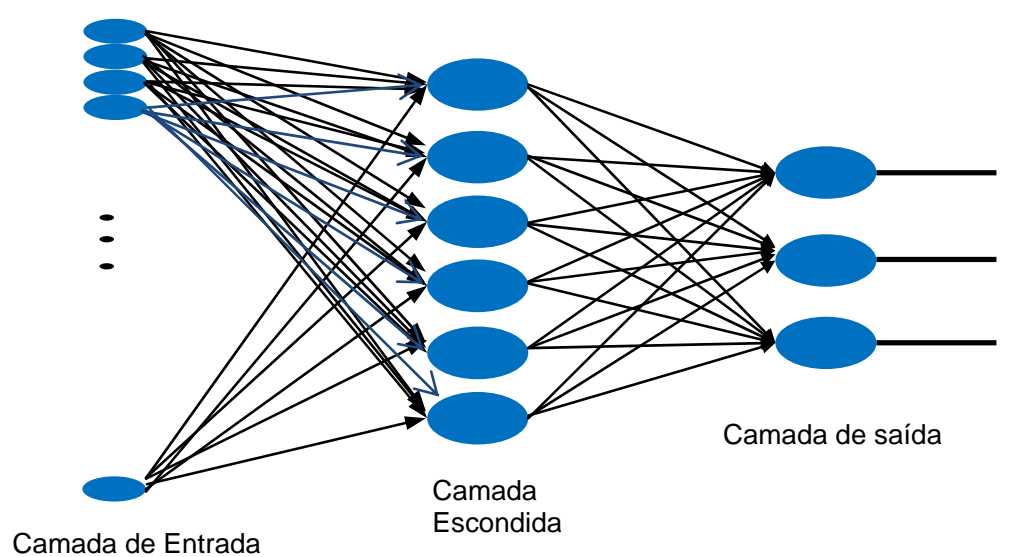

Figura 3: Rede Neural Artificial com 19 entradas, 1 camada escondida e 3 saídas.

No caso deste trabalho a rede está organizada em camada de entrada, uma camada escondida e uma camada de saída, como mostra a Figura 3. Na camada de entrada são utilizados dezenove neurônios, um para cada parâmetro, a camada escondida é composta por seis neurônios e a camada de saída três neurônios para o classificador de três classes e dois neurônios para o classificador de duas classes.

\subsection{Treinamento dos classificadores}

Os classificadores foram desenvolvidos se estimando seus parâmetros (vetores sinápticos e bias) usando o algoritmo de retropropagação do erro [8,9]. Algumas configurações de parâmetros de treinamento foram estudadas para fornecer o melhor resultado possível na distinção das classes e garantir a generalização dos classificadores [1, 2].

\subsection{Estimação da acurácia dos classificadores}

Em reconhecimento de padrões, uma das questões mais polêmicas é saber qual a verdadeira acurácia dos classificadores, isto é, qual o índice de acerto esperado para qualquer conjunto de sinais/dados testado na classificação. Vários conjuntos de dados para treinamento e teste foram sorteados aleatoriamente sem reposição de dados com intuito de estimar a acurácia de identificação dos sinais de Sem Propagação (SP), Propagação Estável (PE) e Propagação Instável (PI) de defeitos, assim como para duas classes (SP e P).

\section{RESULTADOS E DISCUSSÕES}

Inicialmente foram realizados diversos treinamentos para avaliar qual seria a melhor configuração do classificador a ser usada com as entradas apresentadas visando a melhor generalização possível (testes para 
três classes). Foram treinadas redes sem momento, com momento, sem momento e alfa variável e com momento e alfa variável, variando-se de 1 a 10 neurônios na camada intermediária. Os testes foram realizados com taxa de aprendizagem de 0,1 e momento de 0,9, e depois com 0,5 e 0,9, respectivamente [8]. Definido os melhores parâmetros a serem utilizados no treinamento da rede, partiu-se para os testes com sorteio aleatório sem reposição de conjuntos de treinamento (80\%) e teste (20\%) [11]. Foi definido um classificador ótimo com 6 neurônios, sem momento, com valor de alfa inicial igual a 0,05, fator de incremento de alfa de 1,05 e fator de decréscimo de 0,9, alfa máximo de 0,2 (parâmetros ajustados no programa MATLAB).

\subsection{Separação em duas classes (Primeiro ensaio)}

Para este ensaio, foi usinada no corpo de prova uma trinca interna no centro do mesmo com uso de uma retifica manual com disco de corte com raio igual a $12 \mathrm{~mm}$. A geometria do defeito ficou com um formato semi-elíptico com $160 \mathrm{~mm}$ de comprimento, 1,1 mm de largura e 7,33 mm de profundidade.

Analisando o gráfico da Figura 4 a seguir, para primeiramente uma situação de duas classes, os sinais foram separados na classe SP (Sem Propagação) até o tempo de 6868s (181 bar) e classe P (Propagação) entre 6869s e 12837s (233 bar). Resultando em 2207 sinais de SP e 7834 de P.

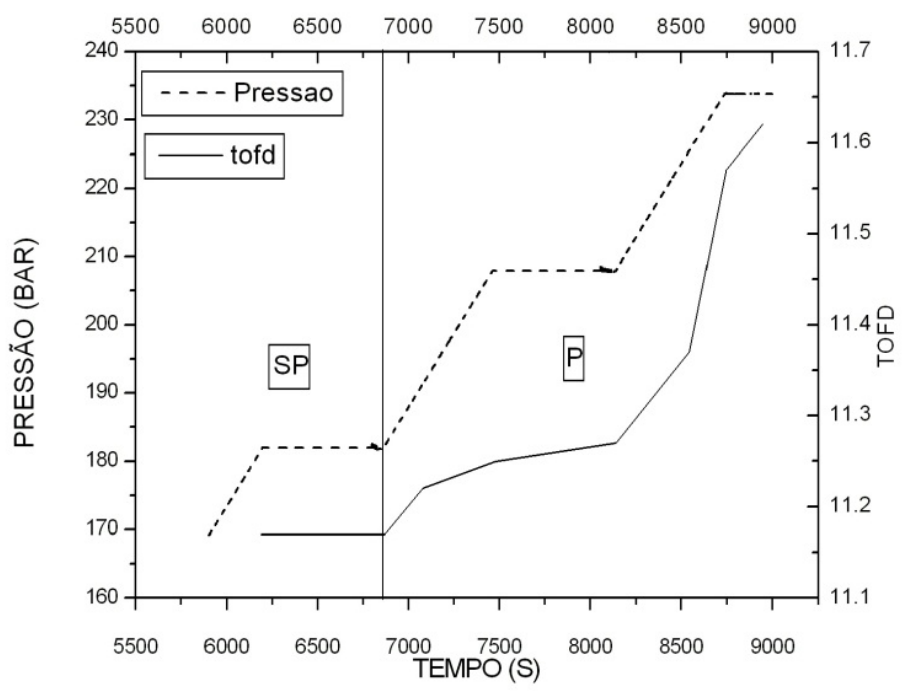

Figura 4: Crescimento do defeito com tempo e pressão de ensaio.

Foram sorteados aleatoriamente 10 pares de conjunto de treinamento e teste (20\% dos sinais pra teste).

A Tabela 2 a seguir apresenta os valores de média de acerto (acurácia de classificação) em quantidade e percentual referentes aos 10 conjuntos sorteados de treinamento e teste. Analisando esta Tabela, os valores de acerto de treinamento são compatíveis com valores já encontrados nos projetos anteriores para discriminação com duas classes [5,12], o mesmo ocorrendo com os sinais usados para testar os classificadores. A pouca diferença entre a média de acerto de treinamento e teste comprova a generalização do classificador, sem provável supertreinamento.

Tabela 2: Resultados da média de acerto para os 10 conjuntos de treinamento e teste (duas classes).

\begin{tabular}{c|c|c|c|c|c}
\hline \multicolumn{2}{c|}{$\begin{array}{c}\text { ACERTOS (CONJUNTOS DE TREINAMENTO) } \\
\text { QUANTIDADE/PERCENTUAL }\end{array}$} & \multicolumn{2}{c}{$\begin{array}{c}\text { ACERTOS (CONJUNTOS DE TESTE) } \\
\text { QUANTIDADE/PERCENTUAL }\end{array}$} \\
\hline & SP & P & & SP & P \\
\hline SP & $920 / 52,17 \%$ & $843 / 47,83 \%$ & SP & $261 / 58,91 \%$ & $182 / 41,09 \%$ \\
\hline P & $235 / 3,74 \%$ & $6035 / 96,26 \%$ & P & $147 / 9,41 \%$ & $1417 / 90,59 \%$ \\
\hline \multicolumn{2}{|c|}{$6955 / 86,58 \%$} & \multicolumn{3}{c}{$1678 / 83,60 \%$} \\
\hline
\end{tabular}




\subsection{Separação em três classes sem reclassificação (primeiro ensaio)}

Para situação de classificação com três classes, analisando a Figura 5, os sinais foram separados na classe SP (Sem Propagação) até o tempo de 6868s (181 bar), na classe PE (Propagação Estável) entre 6869 e 8143 s (208 bar) e na classe PI (Propagação Instável) entre 8144s e 12837s (233 bar). Resultando em 2207 sinais de SP, 1394 sinais de PE e 6439 sinais de PI.

Inicialmente, foram feitos testes com o sistema de classificação considerando apenas o único valor positivo na saída dos neurônios, isto é, o que se denomina sem reclassificação (mais conservador) [6].

Pela Tabela 3 de confusão a seguir, a acurácia de acerto com três classes sem reclassificação atinge $74 \%$ para os conjuntos de treinamento e $71 \%$ com os conjuntos de teste, comprovando a generalização dos classificadores não lineares. Há de se destacar que a configuração da rede neural foi a mesma em todas as situações, conforme explanado anteriormente.

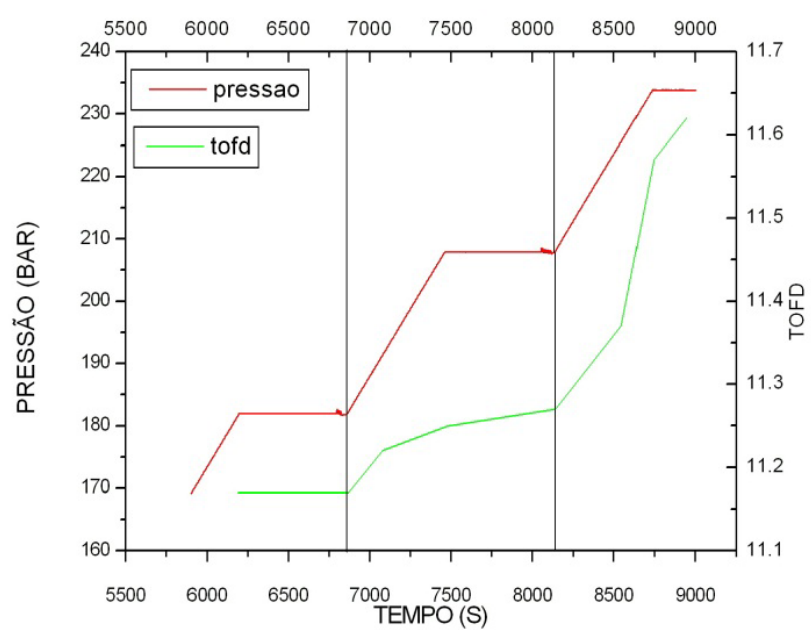

Figura 5: Crescimento do defeito com tempo e pressão de ensaio.

Analisando-se separadamente os índices médios de acerto pra cada uma das três classes agora estudadas, a classe PI é a que tem a melhor acurácia de classificação (95\%), o que era esperado, pois fenomenologicamente é quando há o crescimento instável do defeito e os eventos de emissão acústica se tornam mais perceptíveis no sistema de aquisição. A classe PE confunde bastante com SP e também com PI, o que prova que constatar o exato momento de transição do estado de não propagação do defeito para o estado de uma propagação elástica, estável, assim como a transição do regime elástico para o plástico é consideravelmente mais complexa. Os valores de Não Classificação (mais de uma saída positiva ou todas negativas) estão dentro do esperado no que normalmente acontece em reconhecimento de padrão.

Tabela 3: Resultados da média de acerto para os 10 conjuntos de treinamento e teste (três classes sem reclassificação).

ACERTOS (CONJUNTOS DE TREINAMENTO) QUANTIDADE/PERCENTUAL

\begin{tabular}{c|c|c|c|c}
\hline & SP & PE & PI & NC $^{*}$ \\
\hline SP & $921 / 68,90 \%$ & $54 / 4,02 \%$ & $362 / 27,08 \%$ & \\
\cline { 1 - 3 } PE & $89 / 13,82 \%$ & $289 / 45,20 \%$ & $262 / 40,97 \%$ & \multirow{2}{*}{$157 / 8 \%$} \\
\cline { 1 - 3 } PI & $144 / 2,89 \%$ & $107 / 2,14 \%$ & $4737 / 94,97 \%$ & \\
\hline Média total de acerto & \multicolumn{3}{|c}{$5947 / 74,14 \%$} & \\
\hline
\end{tabular}

ACERTOS (CONJUNTOS DE TESTE) QUANTIDADE/PERCENTUAL

\begin{tabular}{c|c|c|c|c}
\hline & SP & PE & PI & \multirow{2}{*}{ NC $^{*}$} \\
\hline SP & $204 / 51,13 \%$ & $21 / 5,17 \%$ & $174 / 43,70 \%$ & \multirow{2}{*}{$157 / 8 \%$} \\
\cline { 1 - 4 } PE & $28 / 12,66 \%$ & $41 / 18,56 \%$ & $150 / 68,76 \%$ & \\
\cline { 1 - 3 } PI & $33 / 2,67 \%$ & $17 / 1,38 \%$ & $1187 / 95,94 \%$ & \\
\hline
\end{tabular}

NC: Não classificação 


\subsection{Separação em três classes com reclassificação (primeiro ensaio)}

O próximo passo nos testes foi avaliar a classificação dos mesmos pares de conjuntos formados no item anterior no sistema de Reclassificação. Nesta metodologia, lembrando, o neurônio que indica a classe é o de maior valor [6], apontando a maior probabilidade de que um sinal pertença a essa classe.

A Tabela 4 de confusão apresenta os resultados obtidos nesta classificação. As acurácias de treinamento e teste aumentaram em relação à situação anterior, como era de se esperar. Em treinamento a média chegou a quase $83 \%$, e nos testes, 73,54\%, 2,5\% a mais que para Sem Reclassificação. Esta Tabela de confusão também indica que as classes que mais se confundem são PE com SP e PI, ratificando os resultados do item anterior.

Tabela 4: Resultados da média de acerto para os 10 conjuntos de treinamento e teste (três classes com reclassificação).

\begin{tabular}{|c|c|c|c|}
\hline \multicolumn{4}{|c|}{ ACERTOS (CONJUNTOS DE TREINAMENTO) QUANTIDADE/PERCENTUA } \\
\hline & SP & PE & $\mathbf{P I}$ \\
\hline SP & $1149 / 65,54 \%$ & $281 / 16,03 \%$ & $323 / 18,43 \%$ \\
\hline PE & $144 / 12,98 \%$ & $739 / 66,64 \%$ & $226 / 20,38 \%$ \\
\hline $\mathbf{P I}$ & $206 / 3,99 \%$ & $205 / 3,97 \%$ & $4758 / 92,05 \%$ \\
\hline Média total de acerto & \multicolumn{3}{|c|}{$6646 / 82,75 \%$} \\
\hline \multicolumn{4}{|c|}{ ACERTOS (CONJUNTOS DE TESTE) QUANTIDADE/PERCENTUAL } \\
\hline & SP & PE & $\mathbf{P I}$ \\
\hline SP & $219 / 48,34 \%$ & $23 / 5,08 \%$ & $211 / 45,58 \%$ \\
\hline PE & $43 / 15,09 \%$ & $47 / 16,49 \%$ & $195 / 68,42 \%$ \\
\hline $\mathbf{P I}$ & $42 / 3,29 \%$ & $19 / 1,49 \%$ & $1215 / 95,22 \%$ \\
\hline Média total de acerto & \multicolumn{3}{|c|}{$1481 / 73,54 \%$} \\
\hline
\end{tabular}

\subsection{Separação em duas classes (segundo ensaio)}

A geometria e dimensões do corpo de prova e da trinca neste ensaio foram similares ao do ensaio anterior. Analisando o gráfico da Figura 6 a seguir, para primeiramente uma situação de duas classes, os sinais foram separados na classe SP (Sem Propagação) até o tempo de 6740s e classe P (Propagação) a partir de 6741s, resultando em 18908 sinais de SP e 810 de P. Foram sorteados aleatoriamente 10 pares de conjunto de treinamento e teste (20\% para teste).

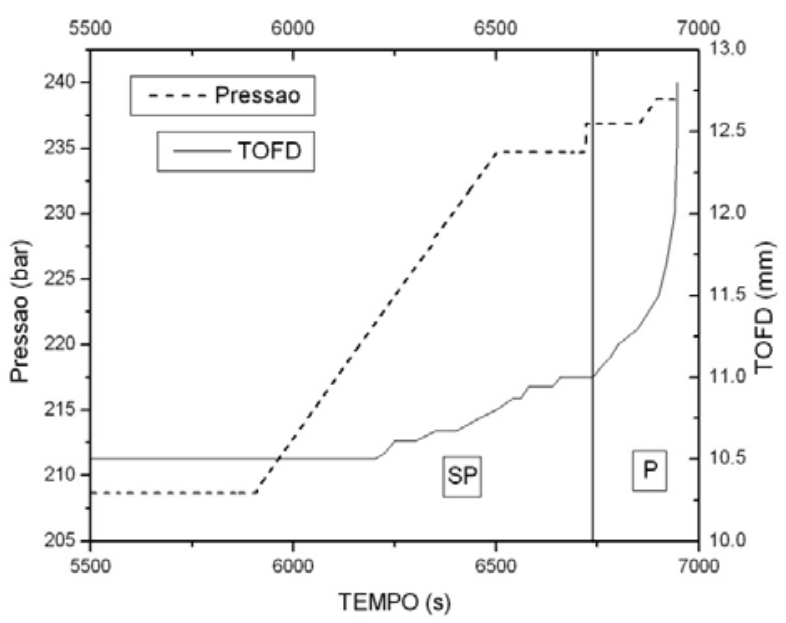

Figura 6: Crescimento do defeito com tempo e pressão de ensaio.

A Tabela 5 a seguir apresenta os valores de média de acerto em quantidade e percentual referentes aos 10 conjuntos sorteados de treinamento e teste.

Na Tabela 5, observam-se excelentes resultados de acurácia de classificação para os sinais de treinamento e teste com valores praticamente iguais, fato que comprova a generalização do classificador não 
linear. Esses números são similares aos obtidos em publicações anteriores $[5,12]$ desenvolvido para uma situação em que se utilizaram todos os parâmetros relacionados, e também quando houve rompimento do corpo de prova. Certamente a provável causa pra estes resultados está no fato dos sinais de EA serem evidenciados no crescimento rápido da trinca até o rompimento (vazamento). Ressalta-se, também, que em relação ao ensaio anterior, a separação das classes SP e P foi realizada se somando os sinais de SP e PE, com PI representando P. Ao contrário, anteriormente os sinais de PE foram somados aos sinais de PI, o que também pode ter corroborado para o índice maior de acerto na atual situação.

Tabela 5: Resultados da média de acerto para os 10 conjuntos de treinamento e teste (duas classes).

\begin{tabular}{c|c|c|c|c|c}
\hline \multicolumn{2}{c|}{$\begin{array}{c}\text { ACERTOS (CONJUNTOS DE TREINAMENTO) } \\
\text { QUANTIDADE/PERCENTUAL }\end{array}$} & \multicolumn{2}{c}{$\begin{array}{c}\text { ACERTOS (CONJUNTOS DE TESTE) } \\
\text { QUANTIDADE/PERCENTUAL }\end{array}$} \\
\hline & SP & P & & SP & P \\
\hline SP & $14909 / 98,53 \%$ & $222 / 1,47 \%$ & SP & $3721 / 98,54 \%$ & $55 / 1,46 \%$ \\
\hline P & $17 / 2,64 \%$ & $628 / 97,36 \%$ & P & $5 / 3,19 \%$ & $161 / 96,81 \%$ \\
\hline Média total de acerto & \multicolumn{2}{|c|}{$15536 / 98,49 \%$} & $3883 / 98,47 \%$ \\
\hline
\end{tabular}

\subsection{Separação em três classes sem reclassificação (segundo ensaio)}

Para situação de classificação com três classes, analisando a Figura 7, os sinais foram separados na classe SP (Sem Propagação) até o tempo de 6200s, na classe PE (Propagação Estável) entre 6201 ate 6740s e na classe PI (Propagação Instável) a partir de 6741s, resultando em 16072 sinais de SP, 2836 sinais de PE e 810 sinais de PI.

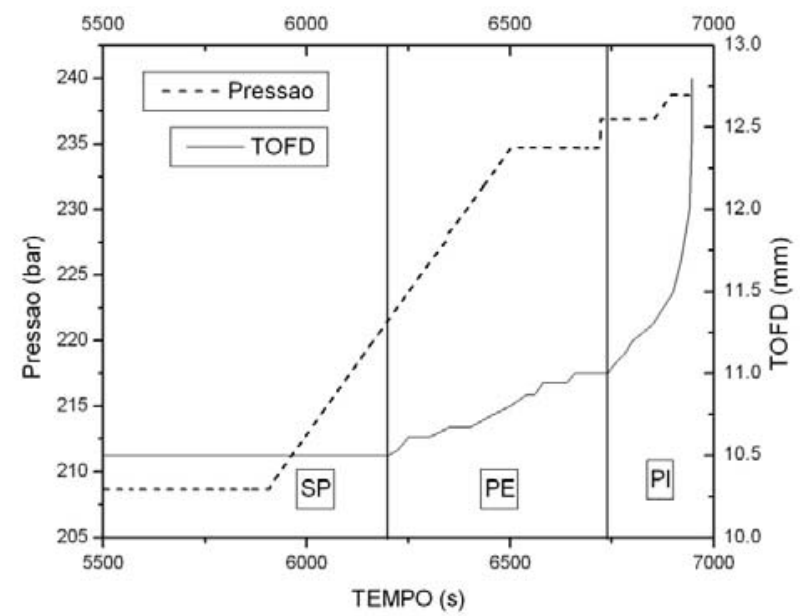

Figura 7: Crescimento do defeito com tempo e pressão de ensaio.

Pela Tabela 6 de confusão a seguir, a acurácia de acerto com três classes Sem Reclassificação atinge cerca de $86 \%$ para os conjuntos de treinamento e teste, comprovando a generalização dos classificadores não lineares. Há de se destacar que a conFiguração da rede neural foi a mesma em todas as situação, conforme explanado anteriormente. Estes valores, comparados aos encontrados no teste anterior, na mesma condição, chegam a ter $15 \%$ acima de acurácia. Uma provável explicação para índice maior de acerto está no fato de ter acontecido um crescimento do defeito de forma rápida até o vazamento do corpo de prova, aumentando claramente o acerto da classe PI. Além disso, as classes SP e PI apresentaram médias de acerto bem maiores que no ensaio anterior, ao contrário de PE que teve uma redução significativa, havendo muita confusão dessa classe com SP, porém, a proporção de sinais desta classe é bem inferior a SP e PI. A quantidade de sinais não classificados é muito pequena para treinamento, apenas 39, e um pouco maior para teste, 175. 
Tabela 6: Resultados da média de acerto para os 10 conjuntos de treinamento e teste (três classes sem reclassificação). NC - não classificado.

\begin{tabular}{|c|c|c|c|c|}
\hline \multicolumn{5}{|c|}{ ACERTOS (CONJUNTOS DE TREINAMENTO) QUANTIDADE/PERCENTUAL } \\
\hline & SP & PE & PI & $\mathrm{NC}^{*}$ \\
\hline SP & $12815 / 99,86 \%$ & $17 / 0,14 \%$ & $1 / 0,01 \%$ & \multirow{4}{*}{$39 / 0,25 \%$} \\
\hline PE & $1864 / 82,63 \%$ & $135 / 5,99 \%$ & $257 / 11,38 \%$ & \\
\hline PI & $0 / 0 \%$ & $13 / 1,93 \%$ & $636 / 98,07 \%$ & \\
\hline Média total de acerto & \multicolumn{3}{|c|}{$13586 / 86,12 \%$} & \\
\hline \multicolumn{5}{|c|}{ ACERTOS (CONJUNTOS DE TESTE) QUANTIDADE/PERCENTUAL } \\
\hline & SP & PE & PI & $\mathrm{NC}^{*}$ \\
\hline SP & $3208 / 99,71 \%$ & $9 / 0,28 \%$ & $0 / 0,01 \%$ & \multirow{4}{*}{$175 / 8,73 \%$} \\
\hline PE & $452 / 81,50 \%$ & $37 / 6,65 \%$ & $66 / 11,86 \%$ & \\
\hline $\mathbf{P I}$ & $0 / 0 \%$ & $3 / 1,68 \%$ & $158 / 98,32 \%$ & \\
\hline Média total de acerto & \multicolumn{3}{|c|}{$3403 / 86,31 \%$} & \\
\hline
\end{tabular}

\subsection{Separação em três classes com reclassificação (segundo ensaio)}

O próximo passo nos testes foi avaliar a classificação dos mesmos pares de conjuntos formados no item anterior no sistema de Reclassificação.

A Tabela 7 de confusão apresenta os resultados obtidos nesta classificação. As acurácias de treinamento e teste aumentaram em relação à situação anterior, porém, de forma insignificante, menos de $1 \%$. Obtiveram-se valores idênticos entre o resultado de treinamento e teste (86,45\%), provando a generalização do processo de classificação.

Tabela 7: Resultados da média de acerto para os 10 conjuntos de treinamento e teste (três classes com reclassificação).

\begin{tabular}{|c|c|c|c|}
\hline \multicolumn{4}{|c|}{ ACERTOS (CONJUNTOS DE TREINAMENTO) QUANTIDADE/PERCENTUAL } \\
\hline & SP & PE & $\mathbf{P I}$ \\
\hline SP & $12824 / 99,80 \%$ & $24 / 0,19 \%$ & $1 / 0,01 \%$ \\
\hline PE & $1871 / 82,52 \%$ & $146 / 6,43 \%$ & $250 / 11,05 \%$ \\
\hline PI & $0 / 0,02 \%$ & $13 / 2,03 \%$ & $637 / 97,95 \%$ \\
\hline Média total de acerto & \multicolumn{3}{|c|}{$13607 / 86,45 \%$} \\
\hline \multicolumn{4}{|c|}{ ACERTOS (CONJUNTOS DE TESTE) QUANTIDADE/PERCENTUAL } \\
\hline & SP & PE & $\mathbf{P I}$ \\
\hline SP & $3211 / 99,63 \%$ & $12 / 0,36 \%$ & $0 / 0,01 \%$ \\
\hline PE & $454 / 81,08 \%$ & $40 / 7,08 \%$ & $66 / 11,84 \%$ \\
\hline PI & $0 / 0 \%$ & $3 / 1,74 \%$ & $158 / 98,26 \%$ \\
\hline Média total de acerto & \multicolumn{3}{|c|}{$3409 / 86,45 \%$} \\
\hline
\end{tabular}

\subsection{Relevância de parâmetros}

Em relação às investigações da relevância dos parâmetros para discriminação das classes de sinais de EA, o gráfico da Figura 8 mostra que AMP e ASL influenciam apenas na classificação de SP e PI, enquanto que o I-Freq e o C-Freq são fundamentais para a discriminação de PE, assim como Freq-PP3. O parâmetro I-Freq contribuiu para a identificação de SP e PE, enquanto que Freq-PP1 e Freq-PP2 para as três classes. Os demais parâmetros contribuem pouco para a discriminação entre as classes. 


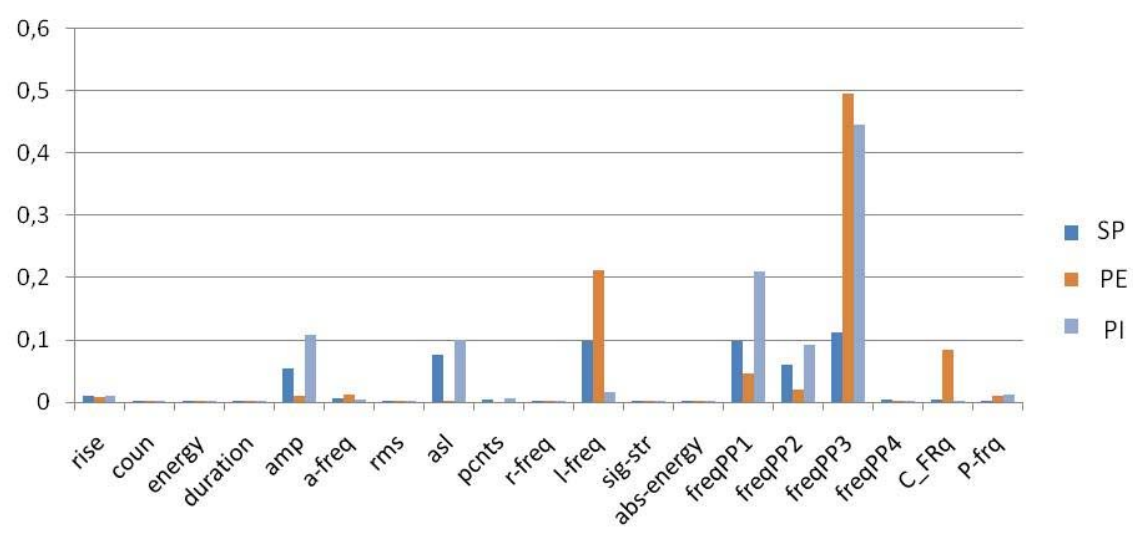

Figura 8: Gráfico da relevância de cada parâmetro para cada uma das três classes.

O gráfico da Figura 9 (valores de relevância multiplicados por 100 para facilitar visualização) mostra que o parâmetro de maior relevância é o Freq - PP3, em seguida os parâmetros Freq-PP1 e I-Freq têm alta relevância, em torno de 30. Os AMP, ASL e o Freq-PP2 apresentam relevância um pouco abaixo de 20 e o C-Freq entre 5 e 10. O demais parâmetros apresentaram valores abaixo de 5 , indicando pouca ou nenhuma influência na classificação.

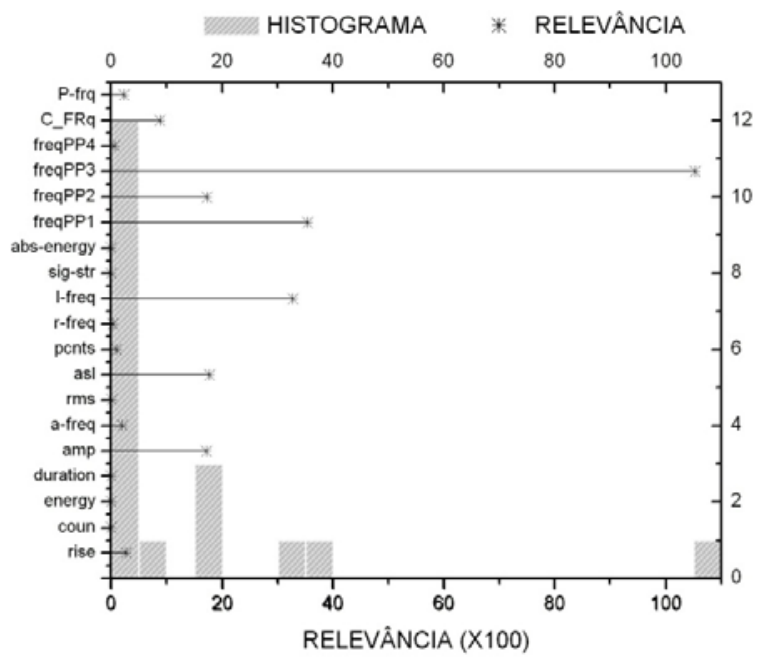

Figura 9: Em cinza o histograma das relevâncias para as três classes juntas. As estrelas indicam a relevância de cada parâmetro para as três classes juntas.

Para confirmar a influência de cada parâmetro na discriminação das classes, criaram-se cinco conjuntos com os pares de entrada e saída, conforme Figura 10. Quanto mais no interior do gráfico, maior a relevância.

Os conjuntos de entrada foram formados da seguinte forma: o valor do parâmetro contido no conjunto foi mantido inalterado e os não contidos no conjunto foram substituídos pelo seu valor médio. Por exemplo, no conjunto 5, os valores de Freq-PP3 foram mantidos, enquanto que os demais parâmetros foram substituídos pela média. Depois de formado, cada conjunto foi testado no classificador.

A Figura 10 indica que a taxa de acerto entre o conjunto 1 e o conjunto 2 é praticamente igual, confirmando que os parâmetros com relevância inferior a 5 não influenciam na classificação. A eliminação de C-Freq, reduziu o acerto em 7\%. O conjunto 4 alcançou quase de 70\%, usando apenas 3 parâmetros, e o conjunto 5, 35\%, com apenas 1 parâmetro. Este resultado confirma o indicado pela Figura 9, que os parâmetros com relevância menor que 5 não influenciam significativamente na classificação. 


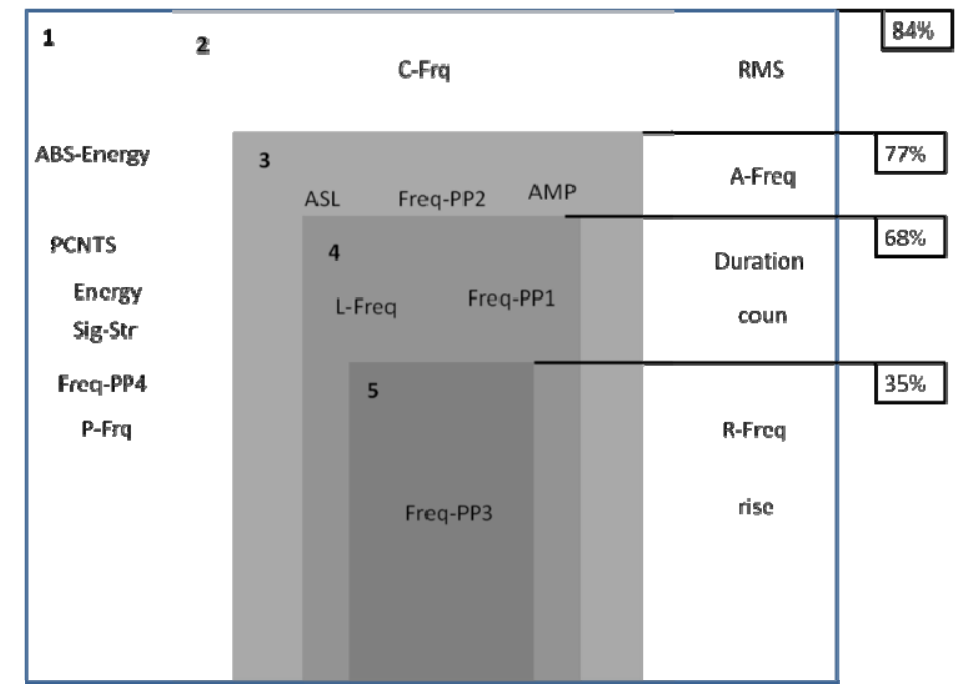

Figura 10: Separação de conjuntos pela relevância. À direita o percentual de acerto na classificação de cada conjunto.

Com objetivo de corroborar os resultados apresentados, uma nova rede foi treinada utilizando os mesmos pares de entrada e saída já utilizados neste item. Contudo, a entrada contém apenas os parâmetros do conjunto 2 da Figura 10, isto é, apenas os sete mais relevantes. A configuração da rede foi a mesma utilizada nos testes anteriores.

As Tabelas 8 e 9 apresentam os resultados obtidos na classificação com a rede treinada com todos os parâmetros e a classificação com a rede treinada com os sete parâmetros mais relevantes (C-Freq, ASL, AMP, I-Freq, Freq-PP1, Freq-PP2, Freq-PP3), respectivamente.

Tabela 8: Tabela confusão da rede treinada com todos os 19 parâmetros de entrada.

\begin{tabular}{|c|c|c|c|}
\hline & SP & PE & PI \\
\hline SP & $1331 / 75,24 \%$ & $210 / 11,87 \%$ & $228 / 12,89 \%$ \\
\hline PE & $203 / 18,27 \%$ & $817 / 73,54 \%$ & $91 / 8,19 \%$ \\
\hline PI & $279 / 5,42 \%$ & $235 / 4,56 \%$ & $4638 / 90,02 \%$ \\
\hline Média total de acerto & \multicolumn{3}{|c|}{$6786 / 84,49 \%$} \\
\hline \multicolumn{4}{|c|}{ ACERTOS (CONJUNTOS DE TESTE) QUANTIDADE/PERCENTUAL } \\
\hline & SP & PE & PI \\
\hline SP & $333 / 76,03 \%$ & $56 / 12,79 \%$ & $49 / 11,19 \%$ \\
\hline PE & $40 / 14,13 \%$ & $217 / 76,68 \%$ & $26 / 9,19 \%$ \\
\hline PI & $71 / 5,52 \%$ & $52 / 4,04 \%$ & $1164 / 90,44 \%$ \\
\hline Média total de acerto & \multicolumn{3}{|c|}{$1714 / 85,36 \%$} \\
\hline
\end{tabular}


Tabela 9: Tabela confusão da rede treinada com os 7 parâmetros mais relevantes (C-Freq, ASL, AMP, IFreq,Freq-PP1, Freq-PP2, Freq-PP3).

\begin{tabular}{|c|c|c|c|}
\hline \multicolumn{4}{|c|}{ ACERTOS (CONJUNTOS DE TREINAMENTO) QUANTIDADE/PERCENTUAL } \\
\hline & SP & PE & $\mathbf{P I}$ \\
\hline SP & $979 / 55,34 \%$ & $418 / 23,63 \%$ & $372 / 21,03 \%$ \\
\hline PE & $122 / 10,98 \%$ & $818 / 73,63 \%$ & $171 / 15,39 \%$ \\
\hline PI & $219 / 4,25 \%$ & $185 / 3,59 \%$ & $4748 / 92,16 \%$ \\
\hline Média total de acerto & \multicolumn{3}{|c|}{$6545 / 81,49 \%$} \\
\hline \multicolumn{4}{|c|}{ ACERTOS (CONJUNTOS DE TESTE) QUANTIDADE/PERCENTUAL } \\
\hline & SP & PE & $\mathbf{P I}$ \\
\hline SP & $301 / 68,72 \%$ & $63 / 14,38 \%$ & $74 / 16,89 \%$ \\
\hline PE & $44 / 15,55 \%$ & $190 / 67,14 \%$ & $49 / 17,31 \%$ \\
\hline $\mathbf{P I}$ & $48 / 3,73 \%$ & $42 / 3,26 \%$ & $1197 / 93,01 \%$ \\
\hline Média total de acerto & \multicolumn{3}{|c|}{$1688 / 84,06 \%$} \\
\hline
\end{tabular}

A rede treinada com os sete parâmetros mais relevantes alcançou um desempenho muito próximo da rede treinada com todos os parâmetros, apresentando uma redução de $4 \%$ na classificação correta no conjunto de treinamento e de apenas $1 \%$ no conjunto de teste. Destaca-se que nestes testes os valores de acerto foram maiores do que de treinamento, o que normalmente não acontece em classificação de padrão, porém, há possibilidade de que isso ocorra com alguns conjuntos de dados.

\section{CONCLUSÕES}

O presente projeto almejava realizar uma série de investigações de separação de classes de sinais de Emissão Acústica para monitoramento de dutos rígidos, dando continuidade aos trabalhos anteriores [referências]. Inovadoramente, os estudos agora foram focados principalmente na tentativa de discriminar as classes Propagação Estável (PE) da classe Propagação Instável (PI). Levando-se em consideração que houve também a realização de testes com duas classes e estudo de relevância de parâmetros, as principais conclusões deste trabalho são:

- Havendo propagação da trinca até o rompimento do corpo de prova, atinge-se quase de $100 \%$ de acerto na separação das classes SP e P, quando o conjunto de sinais contém todos os parâmetros relevantes. Isso reforça estatisticamente os resultados obtidos nos trabalhos anteriores.

- Em relação à relevância dos parâmetros estudados (19 neste trabalho, máximo possível no aparelho de EA empregado), os parâmetros que se mostraram mais eficientes são FRQ PP3, FRQ PP1, IFRQ, FRQ PP2, ASL, AMP e C-FRQ.

- Em relação às classificações com três classes, inovação neste projeto, atinge-se cerca de 86\% de acerto total de classificação quando há propagação até rompimento do corpo de prova, índice considerado significante em função dos poucos parâmetros empregados. Entretanto, a separação entre SP e PE é bem mais complexa que SP e PI e vice-versa, assim como entre PE e PI. Este resultado pode encontrar explicações na pouca diferença que há em termos de fenômeno de Emissão Acústica entre o momento de uma deformação elástica e início de uma deformação plástica. Há de se destacar, porém, que os resultados logrados neste trabalho são bastante relevantes quando comparados aos anteriores, permitindo um desenvolvimento significativo no embasamento necessário de conhecimento para construção de um sistema artificialmente inteligente de detecção de propagação de defeitos em dutos rígidos.

- Os testes com o sistema de Reclassificação, recorrendo a uma forma menos conservadora de classificação de padrões, permitem aumentar os índices de acerto.

\section{TRABALHOS FUTUROS}

Uma sugestão de continuidade de estudo será estudar e viabilizar a formação de novos parâmetros característicos dos sinais, seja por combinação dos atuais em alguma forma de cálculo, ou através da análise 
da forma de onda dos sinais, que foram adquiridas nos últimos ensaios deste projeto pela equipe do Laboratório de Metalurgia Física da UFRGS.

Há também a possibilidade de utilização de técnicas de processamento de sinais, como Análise dos Componentes Principais (PCA) para separar os sinais referentes à propagação do defeito dos demais sinais de outras fontes existentes no corpo de prova durante o ensaio hidrostático.

\section{AGRADECIMENTOS}

Os autores gostariam de agradecer ao CNPQ, a CAPES e FAPERJ, pelo suporte financeiro, ao Laboratório de Processamento de Sinais da COPPE (LPS) pela utilização de seus computadores e programas.

\section{BIBLIOGRAFIA}

[1] Pinto, C.F.C., Silva, R.R., Calôba, L.P., et al., 'Automatic Detection of Defects Propagation in Pipelines: Part I - Pattern Recognition', resultados submetidos.

[2] Pinto, C.F.C, "Monitoração de Defeitos em Dutos Rígidos por Emissão Acústica e Redes Neurais, Dissertação M.Sc., Universidade Federal do Rio de Janeiro, Rio de Janeiro, Brasil, 2011.

[3] Silva R. R, Calôba L. P, Siqueira M. H., et al., "Pattern recognition of weld defects detected by radiographic test”., NDT\&E International, v.37, n.6, 461-470., 2004.

[4] Carvalho, A. A. Silva, R.R. Silva, Rebello, J.M.A., et al., "Pattern Recognition Techniques Applied to the Detection and Classification of Welding Defects by Magnetic Testing”, Research in Nondestructive Evaluation, v.21, n. 2, 91-111, 2010.

[5] Silva, R.R., Soares, S.D., Calôba, L.P., et al., "Detection of the propagation of defects in pressurized pipes by means of the acoustic emission technique using artificial neural networks”. Insight, v.48, n.1, 4551, 2006.

[6] Silva, R. R., Calôba, L. P., Siqueira, M. H., et al., "Evaluation of the relevant characteristic parameters of welding defects and probability of correct classification using linear classifiers”. Insight, v.44, n.10, 616-622, 2002.

[7] J.M. Seixas, L.P. Calôba, Delpino, I., "Relevance Criteria for Variance Selection in Classifier Designs". In: International Conference on Engineering Applications of Neural Networks, pp.451-454, 1996.

[8] Haykin S., Neural Networks - A Comprehensive Foundation., USA, Macmillan College Publishing. Inc., 1994

[9] Duda, R.O., Hart, P.E., Stork, D.G., Pattern Classification, $2^{\text {nd }}$ edition , U.S.A., 2001

[10] T. Hastie, Tibshirani, R., Friedman, J., The Elements of Statistical Learning - Data Mining, Inference, and Prediction, $2^{\text {nd }}$ edition, USA, 2008.

[11] Silva, R. R., Siqueira, M.H.S., Souza, M.P.V., et al., "Estimated accuracy of classification of defects detected in welded joints by radiographic tests". In: NDT \& E International, 38: 335-343, 2005.

[12] Silva, R. R., Mery, D., Soares, S. D., "Evaluation of Acoustic Emission Signal Parameters for Identifying the Propagation of Defects in Pressurized Tubes”, Materials Evaluation, v. 66, p. 493-500, 2008. 